\author{
Who Needs Privacy? \\ Tobias Dienlin ${ }^{1} \&$ Miriam Metzger ${ }^{2}$ \\ ${ }^{1}$ University of Hohenheim \\ ${ }^{2}$ University of California, Santa Barbara
}

Author Note

Tobias Dienlin, Department of Media Psychology, University of Hohenheim, Germany; Miriam Metzger, Department of Communication, University of California, Santa Barbara, United States of America.

Correspondence concerning this article should be addressed to Tobias Dienlin, University of Hohenheim, Department of Media Psychology (540F), 70599 Stuttgart, Germany. E-mail: tobias.dienlin@uni-hohenheim.de 
Abstract

This study analyzes how personality traits relate to need for privacy. We focus on three dimensions: (a) need for privacy from government surveillance, (b) need for privacy from other individuals, and (c) need for anonymity. Using an online questionnaire with 273 student respondents, several significant relationships were found. For example, results showed that less sociable people desired considerably more privacy on all three dimensions. Somewhat more controversially, people who reported less integrity also desired more anonymity. While anxious people desired slightly less privacy from government surveillance, risk avoidant people desired considerably more privacy from other people. Traditionalism did not relate to need for privacy. Together, the results have several implications. For example, they shed light on the "nothing-to-hide argument": A person who desires more anonymity might indeed be of less integrity. The most important predictor of need for privacy, however, was sociability. Indeed, this study is the first to show empirically that if a person desires more privacy, it is most plausible that he or she is less sociable. Keywords: Privacy, need for privacy, personality, anonymity, integrity, SEM 
Who Needs Privacy?

In light of the increasing digitization of everyday life, which has led to several sweeping societal changes such as the commodification and monetization of personal information (Sevignani, 2016), privacy has become a major topic of societal interest. Despite its importance, however, we still know surprisingly little about the relation between privacy and personality (e.g., Masur, 2018, p. 155). Why do some people feel they need or desire more privacy than others do, and how do these people differ from one another? We think that a better understanding of the relation between personality and privacy is crucial: Several theories argue that personality determines privacy behaviors (e.g., Masur, 2018), however, to date there is almost no empirical research that can be used to deduce well-informed hypotheses. As a result, the main question of this paper is: What are personality facets that can be used to best explain peoples' felt need for privacy?

\section{The Need for Privacy}

Privacy as a theoretical concept is both complicated and contested (Nissenbaum, 2010, p. 71). Hence, we begin by outlining our own understanding of privacy. First and foremost, privacy captures the extent of voluntary withdrawal from others (Westin, 1967). Several models suggest that privacy is multi-dimensional. For example, in a theory-driven treatise Burgoon (1982) argued that privacy has four dimensions: informational, social, psychological, and physical privacy. Pedersen (1979), by contrast, argues that on the basis of an empirical factor analysis (initially starting with 94 items) privacy exists on six dimensions: reserve, isolation, solitude, intimacy with friends, intimacy with family, and anonymity. In addition, Schwartz (1968) differentiated between horizontal and vertical privacy; whereas horizontal privacy captures withdrawal from peers, vertical privacy addresses withdrawal from superiors or institutions (e.g., government agencies). The need for privacy, then, captures "[a]n individual's need to selectively control the access of others to the individual self with the aim of achieving a desired level of physical or psychological 
privacy [...]") (Trepte \& Masur, 2017, p. 1). Finally, one can also distinguish between the objective privacy context (e.g., how many users can actually read a post on an SNS?), subjective privacy perceptions (e.g., how many users people think can read a post on an SNS?), and resulting privacy behaviors (e.g., how much do people self-disclose?) (Dienlin, 2014).

For the purpose of this study, we combine the aforementioned notions and focus on (a) vertical privacy with regard to people's felt need for withdrawal from government surveillance, (b) horizontal privacy in terms of the perceived need for withdrawal from peers, friends, or acquaintances, and (c) both horizontal and vertical privacy as captured by people's general felt need for anonymity.

In order to guide the selection of personality dimensions that might explain need for privacy best, it is useful to determine why people actually feel they need privacy. According to Trepte and Masur (2017), the need for privacy is a secondary need - it is not an end in itself, but rather a way to satisfy other more fundamental needs (e.g., time to oneself). Specifically, Westin (1967) defined four ultimate purposes of privacy: (1) self-development (i.e., the integration of experiences into meaningful patterns), (2) autonomy (i.e., the desire to avoid being manipulated and dominated), (3) emotional release (i.e., the release of tension from social role demands), and (4) protected communication (i.e., the ability to foster intimate relationships). Not least, privacy facilitates self-disclosure (Dienlin, 2014), which is vital for attaining social support, initiating relationships, and getting close to other people (Omarzu, 2000).

On the other hand, however, there are negative aspects of privacy too. For example, too much privacy can be problematic. Because human beings are inherently social, being overly cut-off from others can impede flourishing, nurture deviant behavior, or introduce power asymmetries (Altman, 1975). Also, and as mentioned above, privacy fosters self-disclosure, which presents a potential risk because others might disagree, disapprove, or misuse the information in other contexts (Petronio, 2010). The dialectical tension between 
the positive and negative aspects of privacy likely causes variability across individuals in their need for privacy. This study asks: What role does personality play in determining individual-level variations in need for privacy?

\section{Predicting the Need for Privacy}

In what follows we now explore several personality aspects that we think might predict the need for privacy. As there is no established theory that dovetails privacy and personality, it is difficult to deduce precise and well-informed hypotheses. The rationales that we outline below should hence be considered tentative suggestions toward a theory of personality and privacy. Also, please note that we elaborate only on those dimensions of need for privacy for which plausible rationales could be formed.

The general underlying theoretical framework that guided our selection process was the Big Five approach (e.g., John \& Srivastava, 1999). Note that privacy concerns, a variable that is conceptually close to need for privacy, shows only negligible relations with the Big Five factors (Bansal, Zahedi, \& Gefen, 2010; Junglas, Johnson, \& Spitzmüller, 2008). In order to be more precise, we hence follow the advice by Paunonen and Ashton (2001) and refer to specific personality facets instead of generic personality factors. To illustrate, instead of dwelling on the general factor of Extraversion, we instead focused on the facet Gregariousness, which we consider to be more pertinent.

Our reasoning further was guided by another central theoretical tenet. As suggested above, privacy can be either positive or negative. Similarly, other people, the government, and anonymity can be considered either a resource or a threat. Having information about a person's personality can inform us whether he or she is more likely to think of others as a resource or a threat. It follows that if other people are considered a threat it seems to be more likely that a person will desire more privacy from others, and vice versa (Altman, 1976). 
Sociability. First, we argue that need for privacy should be closely related to a person's sociability or gregariousness (which is a subdimension of extraversion, Costa \& McCrae, 1992). Sociability captures whether people prefer to spend their time alone or with company. It seems plausible that people who are more sociable are also more likely to think of other people as a resource, which is why they should generally desire less interpersonal privacy and less anonymity (e.g., Buss, 2001). Put differently, given that privacy is a voluntary withdrawal from society (Westin, 1967), we expect that people who are less sociable, more reserved, or more shy should have a greater need for privacy from others.

This rationale is supported by several empirical studies: People who score higher on the personality meta-factor plasticity, which is a composite of the two personality factors extraversion and openness, have been shown to desire less privacy (Morton, 2013); people who describe themselves as introverted thinkers are more likely to prefer social isolation (Pedersen, 1982); and introverted people are more likely to report invasions of privacy (Stone, 1986).

Integrity. More controversially, it has been argued by some that people need privacy because they have something to hide. The so-called nothing-to-hide argument states that "If you have nothing to hide, you have nothing to fear." As described by Solove, the nothing-to-hide argument says that data mining and surveillance by government entities "is not likely to be threatening to the privacy of law-abiding citizens. Only those who are engaged in illegal activities have a reason to hide this information" (Solove, 2007, p. 753). Hence, another potential predictor of why people need privacy could also be a so-called "lack" of integrity.

Because integrity is a delicate concept, let us first try to define it conceptually. Although in terms of a scientific definition there is no consensus, most scholars seem to agree that integrity "incorporates a tendency to comply with social norms, avoid deviant behavior, and embrace a sense of justice, truthfulness, and fairness" (Connelly, Lilienfeld, 
\& Schmeelk, 2006, p. 82). In order to sidestep the (very legitimate) philosophical debates about what constitutes integrity and what not, we hence follow Paunonen (2002) and adopt a lowest common denominator definition, which means that we only consider participating in explicitly socially-sanctioned or illegal activities as a sign of lack of integrity.

It is possible to think of theoretical arguments for why lack of integrity might correlate positively with a person's felt need for privacy. People who actually commit crimes may face even greater risk from self-disclosure compared to others, because government agencies and people would surely disapprove of their activities (Petronio, 2010). Hence, the government and other people are more likely to be perceived as a threat, which should render anonymity a resource. As a consequence, people with lower integrity might desire more privacy as a means to mitigate their felt risk (Altman, 1976).

There are also a few empirical studies that imply — at least indirectly - a relation between privacy and integrity. For example, studies have found that surveillance can reduce cheating behaviors (Corcoran \& Rotter, 1987; Covey, Saladin, \& Killen, 1989). Covey et al. (1989) for example asked students to solve an impossible maze. In the high surveillance condition, the experimenter stood in front of the students and closely monitored their behavior. In the low surveillance condition, the experimenter remained behind the students where he or she could not see the students. Results showed greater cheating among students in the low surveillance condition, suggesting that in situations with less privacy, people show more integrity (i.e., fewer cheating behaviors). Next, in a longitudinal sample with 457 respondents in Germany (Trepte, Dienlin, \& Reinecke, 2013), people who felt they needed more privacy were also less authentic on their online social network profiles $(r=-.48)$ and less authentic in their personal relationships $(r=-.28)$. Given the argument that authenticity is a subset of integrity (Sheldon, 2004), one could hence also reason that the concept of integrity might relate to a person's perceived need for privacy. Somewhat related, it has been found that people who are more agreeable are also moderately less concerned about their privacy (Junglas et al., 2008). Finally, Pedersen 
(1982) showed that three dimensions of need for privacy relate to self-esteem: Respondents who held a lower self-esteem were more reserved $(r=.29)$, needed more anonymity $(r=$ $.21)$, and preferred solitude $(r=.24)$. While self-esteem and integrity are distinct concepts, Pedersen's specific operationalization of self-esteem integrated several aspects of integrity by using items such as moral, nice, fair, unselfish, good, honest, and reputable (p. 12). In conclusion, we could imagine that lack of integrity may indeed relate to an increased felt need for privacy from government surveillance, as governments have the legitimate power to prosecute illegal activities. Next, it seems plausible that lack of integrity may relate to an increased need for anonymity, as anonymity makes it more difficult for both government and social agents to identify and address potential wrongdoers. Finally, lack of integrity may also relate to an increased need for privacy from other people, as most other people will disapprove of immoral or illegal activities, and could even reveal those activities to authorities.

Anxiety. Next, it seems possible that the need for privacy is also related to people's level of general anxiety (which is a subdimension of neuroticism, Costa \& McCrae, 1992). General anxiety measures for example whether people are afraid of negative events or whether they are easily frightened. With regard to interpersonal privacy, one could argue that people who are anxious are more likely to feel that social interactions pose a greater risk and threat (especially with strangers or weak ties; Granovetter, 1973), which is why anxious people might desire more privacy. Somewhat related, prior empirical research has shown that people who are more concerned about their privacy are also more likely to withdraw online, for example by deleting posts or untagging themselves from linked content (Dienlin \& Metzger, 2016). On the other hand, one could argue in favor of the opposite: People who are more anxious may desire less privacy from others (especially their strong ties), as a means to cope better with their daily challenges.

Concerning the need for privacy from government surveillance, we could imagine that people who are more anxious desire less privacy. Despite the fact that only $18 \%$ of all 
Americans trust their government "to do what is right" (Pew Research Center, 2017), almost everyone agrees that "it's the government's job to keep the country safe," with most people also being satisfied with the government's job (Pew Research Center, 2015). Hence, for anxious individuals, the government might be seen as a resource rather than a threat. It therefore seems plausible that people who are in general more anxious are also more likely to consent to government surveillance, given that such surveillance promises to prevent crime or to reduce the likelihood of terrorist attacks (Greenwald, 2013), implying that people who are more anxious might desire less privacy from government surveillance and, for the same reasons, also less anonymity.

Risk avoidance. Similarly, it could be that an individual's felt need for privacy is related to a general tendency to avoid risks, to deliberate, and to plan carefully (deliberation is a subdimension of conscientiousness, Costa \& McCrae, 1992). Risk avoidance captures the degree to which people prefer to abstain from taking risks. If other people are considered a threat, people who are risk averse should desire more interpersonal privacy, because they may feel greater risk associated with disclosure of personal information. The most cautious strategy to minimize risks of information disclosure would be, arguably, to keep as much information as possible private. Somewhat related, empirical studies report that people who think that their privacy is at risk are less likely to disclose information online (e.g., Bol et al., 2018). Moreover, research suggests that conscientious people are slightly more concerned about their privacy (Junglas et al., 2008). But as above, especially with regard to privacy from government surveillance, risk averse people could also desire less privacy, in order for the government to be able to avert potential threats. In sum, think that it is most plausible that people who are more risk averse also desire more privacy in all three contexts measured in this study.

Traditionality. Next, it seems plausible that need for privacy is also related to traditionality (which is a subdimension of openness to experience, Costa \& McCrae, 1992). Traditionalism measures whether people prefer to stick with their usual routines. 
Computers and the Internet have rendered the world increasingly knowable: Social interactions, purchases, and medical treatments nowadays all produce digital traces, which can be combined into accurate latent user profiles. Given that digital information is persistent, searchable, reproducible, and scalable (boyd, 2008), this allows for unprecedented means and degrees of surveillance. Mark Zuckerberg famously observed that privacy is no longer a social norm, rather the norm is that people share personal information (Johnson, 2010). Hence, in order to be part of contemporary life, it seems necessary to give up some privacy. However, people may not be equally willing to pay that price, and especially people who are more traditional might decide against giving up their privacy.

Empirical research does find that older people, who are generally less open and more traditional (Donnellan \& Lucas, 2008), are more concerned about their privacy (Fife \& Orjuel, 2012). On the other hand, Junglas et al. (2008) report that openness to experience is positively related to privacy concern, which would argue in favor of the exact opposite pattern of results. Taken together, we still consider it plausible that people who are more traditional also desire more privacy in all three contexts measured in this study.

Socio-demographic variables. Finally, it has often been shown that socio-demographic variables such as sex, age, and affluence can relate to the need for privacy (Park, 2015; Tifferet, 2019; Trepte et al., 2013; Weinberger, Zhitomirsky-Geffet, \& Bouhnik, 2017). Although these variables do not specifically address our research question at hand they will be included as control variables - not least as their omission could lead to spurious results or an inflation of false positives.

\section{Method}

This section describes how we determined the sample size, data exclusions, the analyses, and all measures in the study. 


\section{Sample}

Participants were students from an IRB-approved subject pool at a university in the western U.S. All students received course credit for participating in the study. The initial sample consisted of 295 respondents. We removed two cases with problematic response patterns (e.g., straight-lining, missing of inverted items), which we identified using the Guttman value (Meijer, Niessen, \& Tendeiro, 2016). Next, we excluded one participant who provided an illogical age (i.e., 9 years). We also excluded 19 respondents because they answered less than $50 \%$ of all questions. The remaining missing responses $(0.40 \%)$ were imputed using predictive mean matching. Analyses showed no respondents needed to be excluded due to "speeding" (i.e., < 5 min answer time). The final sample consisted of 273 respondents.

Age of participants ranged from 18 to 56 years ( $M=20$ years), with $27 \%$ of the respondents being male. The median participation time was $24.98 \mathrm{~min}$.

The data were collected in 2015. While we aimed to collect a large number of participants (i.e., $N>300$ ), at the time we were not yet aware of the importance to run a-priori power analyses to determine sample size. A post-hoc power analysis shows that the final sample size allowed to find effects with a size of $\beta=.22$ in $95 \%$ of all cases, and that we had power to detect small effects (i.e., $\beta=.10$ ) in $38 \%$ of all cases.

\section{Data Analyses}

All hypotheses were tested using a two-tailed significance level of 5\%. Because all predictors were chosen on the basis of a-priori reasoning we did not control for alpha error inflation. We classified regression coefficients with values exceeding $|\beta|=.10$ as small effects, $|\beta|=.30$ as medium effects, and $|\beta|=.50$ as large effects. Our smallest effect size of interest (SESOI; Lakens, Scheel, \& Isager, 2018) was $|\beta|=.10$. Effects below the SESOI were considered too small to be theoretically relevant.

The factorial validity of the measures and the hypotheses were tested with structural 
equation modeling (SEM). Mardia's test showed that the assumption of multivariate normality was violated, $p$ (skewness) $<.001, p$ (kurtosis) $<.001$. As a result, we used the more robust Satorra-Bentler scaled and mean-adjusted test statistic (MLM) as estimator. Fit was assessed using the conventional measures and criteria as proposed by Kline (2016).

First, we tested the factorial validity of the variables we collected. In a first step, we ran confirmatory factor analyses (CFAs) to determine whether the variables were unidimensional. If fit was not adequate, we then ran exploratory factor analyses (EFAs) to assess the actual underlying factor structure. EFAs were run using maximum likelihood estimation and oblimin rotation (Costello \& Osborne, 2005, p. 7). If more than one dimension was revealed, we tried to find a bifactor model solution. ${ }^{1}$ This way we were able to attain a general measure of the predictors, instead of needing to further complicate our analyses by including subdimensions of the predictors. ${ }^{2}$ If no adequate bifactor model could be found, we proceeded by deleting items with low loadings on the general factor and/or the specific factors. If also after deletion of individual items no bifactor solution emerged, we then aimed to extract a single factor with sufficient factorial validity using a subset of the items.

To anwers our research question, we first had a look at the variables' bivariate relations (see Figure 1). To better assess the variables' potential causal relations, we next ran a multivariate structural regression model. Because we were interested in a complex model (overall, eight predictors and three outcomes) but the sample was comparatively small, we simplified the model. To this end, instead of a fully latent structural regression model we conducted a partially latent structural regression model, in which the predictor

${ }^{1}$ Bifactor models implement one factor that explains the variance in all items (the so-called general factor or g-factor). In addition, at least another two more factors are implemented that explain the variance in a subset of the items. The general factor and the specific factors are orthogonal. Bifactor models are nested within hierarchical models. For more information on bifactor models, see Kline (2016), p. 319.

${ }^{2}$ Note that we did not specify a bifactor model of need for privacy, because we were explicitly interested in the relations between the personality facets and the three dimensions of need for privacy. 
variables were modelled as single indicators while controlling for measurement error (Kline, 2016, p. 214). To get high-quality single indicators of the predictors, we computed the average of the model predicted values / latent factor scores, which we extracted from the CFAs. If the CFAs showed a unidimensional solution, we used the model predicted values for this latent factor; if the CFAs produced a multidimensional solution, we used the model predicted values for the general latent factor (again, by using this procedure we were able to attain a general measure of the predictors, instead of needing to further complicate analyses by including subdimensions of the predictors.) As the main variable of interest, the outcome need for privacy was measured as a three-dimensional latent factor consisting of overall 12 items (see below).

Finally, combining several items into a latent factors helps to reduce and condense information. At the same time, however, this approach also introduces several researcher degrees of freedom. We hence emphasize that we deleted items only in order to achieve satisfactory factorial validity and not in order to cherry-pick significant material. In light of our not having preregistered the analyses and in order to provide the complete picture, in the online supplementary material (OSM) we hence also report how the independent variables predicted each item individually.

R (Version 3.5.1; R Core Team, 2018) and the R-packages GGally (Version 1.4.0; Schloerke et al., 2018), ggplot2 (Version 3.1.0; Wickham, 2016), knitr (Version 1.21; Xie, 2015), lavaan (Version 0.6.3; Rosseel, 2012), mice (Version 3.3.0; van Buuren \& Groothuis-Oudshoorn, 2011), MVN (Version 5.6; Korkmaz, Goksuluk, \& Zararsiz, 2014), papaja (Version 0.1.0.9842; Aust \& Barth, 2018), PerFit (Version 1.4.3; Tendeiro, Meijer, \& Niessen, 2016), psych (Version 1.8.12; Revelle, 2018), pwr (Version 1.2.2; Champely, 2018), sem Tools (Version 0.5.1; Jorgensen et al., 2018), and tidyverse (Version 1.2.1; Wickham, 2017) were used for the analyses. 
Table 1

Psychometrics of Variables Used.

\begin{tabular}{lcccccccccccc}
\hline & $\mathrm{m}$ & $\mathrm{sd}$ & chisq & $\mathrm{df}$ & pvalue & cfi & tli & rmsea & srmr & omega & alpha & ave \\
\hline Privacy need & & & & & & & & & & & & \\
$\quad$ (Combined) & 4.18 & 1.62 & 101.72 & 50 & $<.001$ & .94 & .92 & .06 & .06 & .85 & .88 & .46 \\
Government & 4.13 & 1.49 & 7.26 & 5 & .202 & 1.00 & .99 & .04 & .02 & .87 & .87 & .57 \\
Interpersonal & 4.21 & 1.59 & 10.22 & 2 & .006 & .93 & .78 & .12 & .04 & .62 & .62 & .30 \\
Anonymity & 2.92 & 1.50 & 3.16 & 2 & .206 & .99 & .98 & .05 & .03 & .69 & .70 & .37 \\
Sociability & 4.70 & 1.50 & 6.20 & 2 & .045 & .98 & .94 & .09 & .03 & .79 & .79 & .49 \\
Integrity & 4.56 & 1.84 & 47.44 & 25 & .004 & .96 & .94 & .06 & .04 & .79 & .83 & .41 \\
Anxiety & 4.41 & 1.53 & 36.98 & 18 & .005 & .96 & .93 & .06 & .04 & .80 & .82 & .42 \\
Risk avoidance & 4.30 & 1.56 & 5.27 & 5 & .384 & 1.00 & 1.00 & .01 & .02 & .79 & .79 & .43 \\
Traditionality & 3.91 & 1.60 & 10.69 & 5 & .058 & .98 & .95 & .06 & .03 & .74 & .74 & .37 \\
\hline
\end{tabular}

Note. All items were measured on 7-point scales with Likert response options. Alpha $=$ Cronbach's alpha (internal consistency); omega = Raykov's omega (composite reliability); ave $=$ average variance extracted.

\section{Measures}

In this section we present how we operationalized our constructs. All items were answered on a 7-point Likert scale ranging from 1 (strongly disagree) to 7 (strongly agree). The results of the CFAs showed that it was possible to model all variables with at least acceptable fit. For an overview of the items' psychometrics, factorial validity, and reliability, see Table 1. A list of all the items that were used (including deleted ones), results of CFAs/EFAs, as well as item statistics and their distribution plots can be found in the OSM.

Need for privacy. As the main variable of interest, we collected several items to measure need for privacy. First, we collected four items capturing the general need for 
Table 2

Overview of Items Measuring Need for Privacy

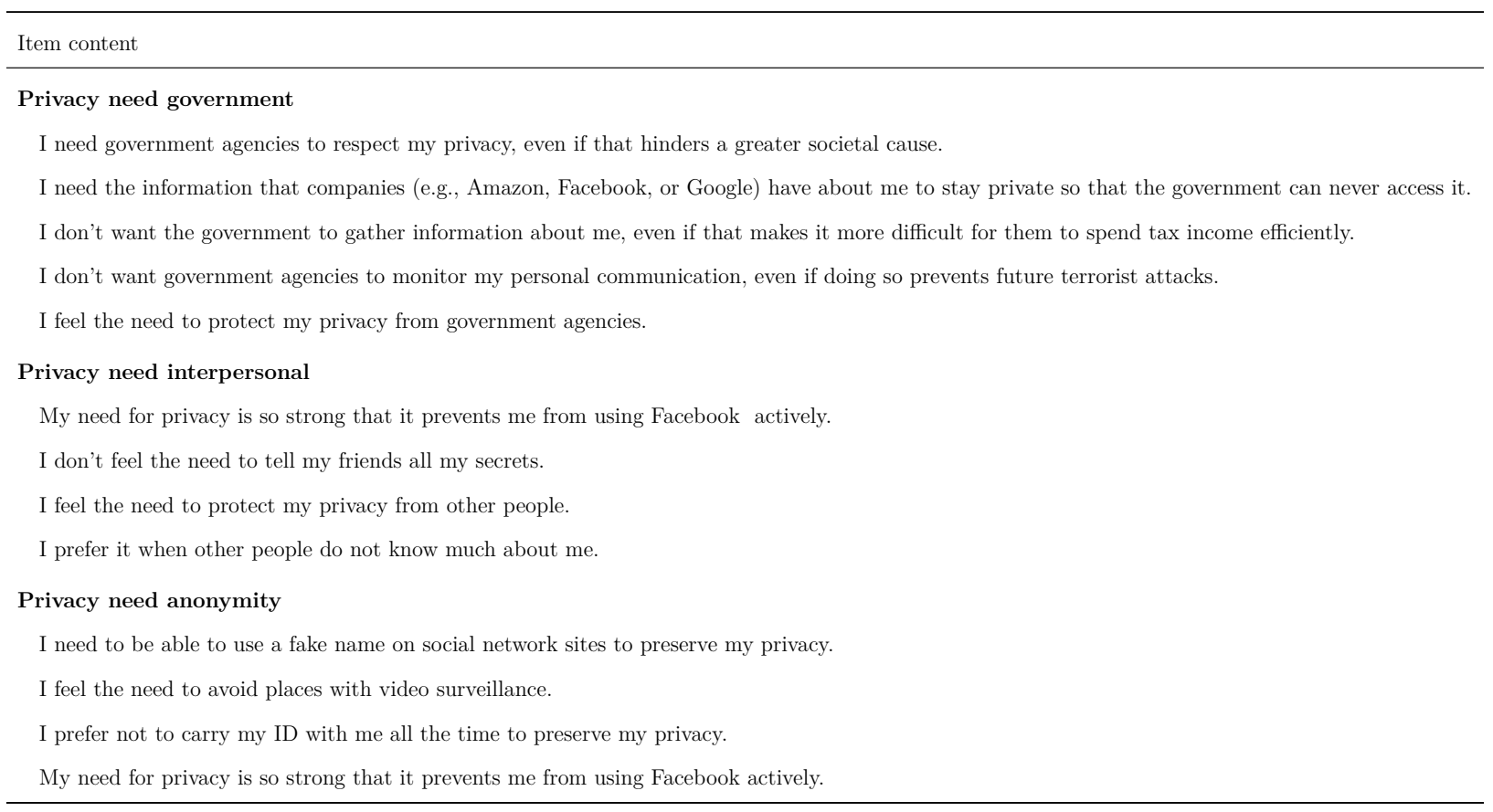

privacy using the subscale information developed by Trepte and Masur (2017). Second, we measured need for privacy on a societal level using nine self-designed items. The first subdimension was government surveillance, which represents the extent to which people want the government to abstain from collecting information about them. The second dimension was anonymity, which captures the extent to which people feel the need to avoid identification. Third, we measured need for privacy on an interpersonal level using nine self-designed items. The first subdimension measures need for privacy from other people in online contexts. The second subdimension measures need for privacy from other people in offline contexts.

EFAs and CFAs revealed a well-fitting model with 12 items (one double-loading), which consisted of the three dimensions (a) need for privacy from the government, (b) need for privacy from other people, and (c) need for anonymity. For a list of all items, see Table 2 . 
Sociability. Sociability was measured with the Extraversion subscale Gregariousness (Costa \& McCrae, 1992), which consists of eight items. An example item is "I shy away from crowds of people." Analyses revealed that a bifactor model based on a subset of six items showed good fit to the data.

Integrity. To measure integrity, we used the subscale Integrity of the Supernumerary Personality Inventory (Paunonen, 2002), which consists of eight items. In addition, we designed another three items. An example item is "I don't think there's anything wrong with cheating a little on one's income tax forms." Analyses revealed that a bifactor model based on a subset of nine items showed good fit to the data.

Anxiety. Anxiety was measured with the Neuroticism subscale Worry (Costa \& McCrae, 1992), which consists of eight items. An example item is "I am easily frightened." Analyses revealed that a bifactor model using all eight items showed good fit to the data.

Risk avoidance. Risk avoidance was measured with the Conscientiousness subscale Deliberation (Costa \& McCrae, 1992), which consists of eight items. An example item is "I plan ahead carefully when I go on a trip." Analyses revealed that a bifactor model using all eight items showed good fit to the data.

Traditionalism. Traditionalism was measured with the (inverted) Openness to Experiences subscale Actions (Costa \& McCrae, 1992), which consists of eight items. An example item is "I'm pretty set in my ways." Analyses revealed that a model with a single factor based on a subset of five items showed good fit to the data.

\section{Results}

For an overview of the results of the bivariate relations, see Figure 1. For the statistical results of the SEM, see Table 3, and for a graphical visualization, see Figure 2. Note that the final structural regression model that was estimated fit the data comparatively well, $\chi^{2}(137)=226.97, p<.001$, cfi $=.92$, rmsea $=.05,90 \%$ CI $[.04, .06]$, $\mathrm{srmr}=.06$. Although fit was not perfect, we decided against further modifications of the 
model in order to avoid overfitting.
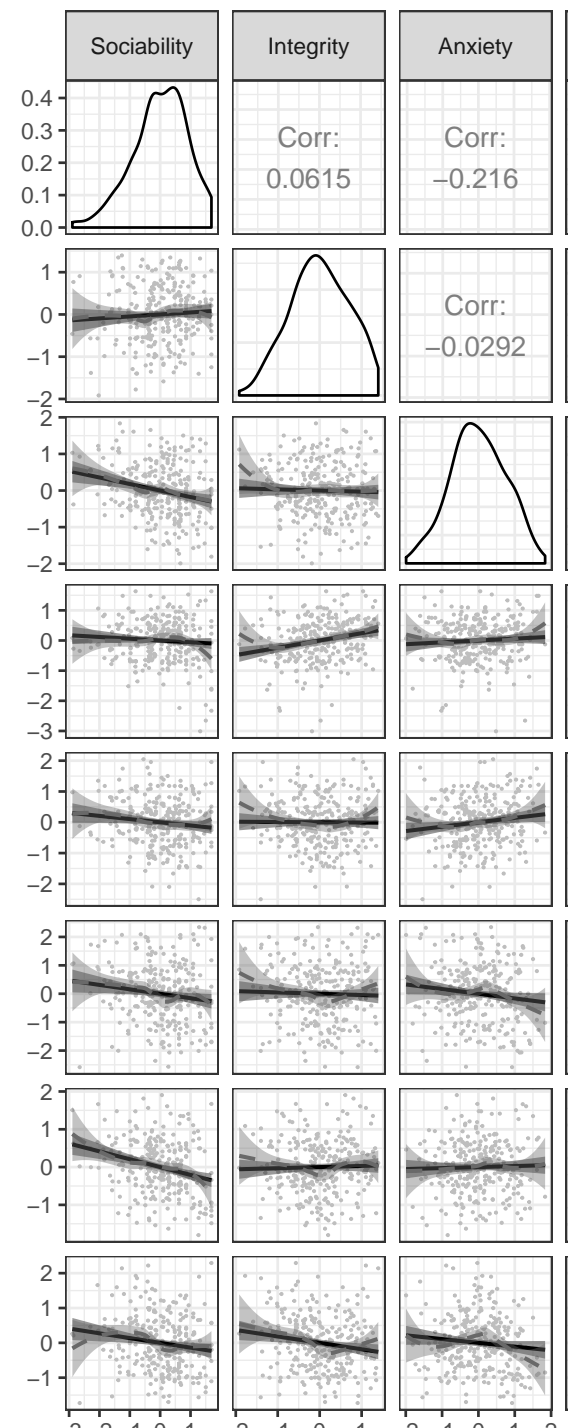
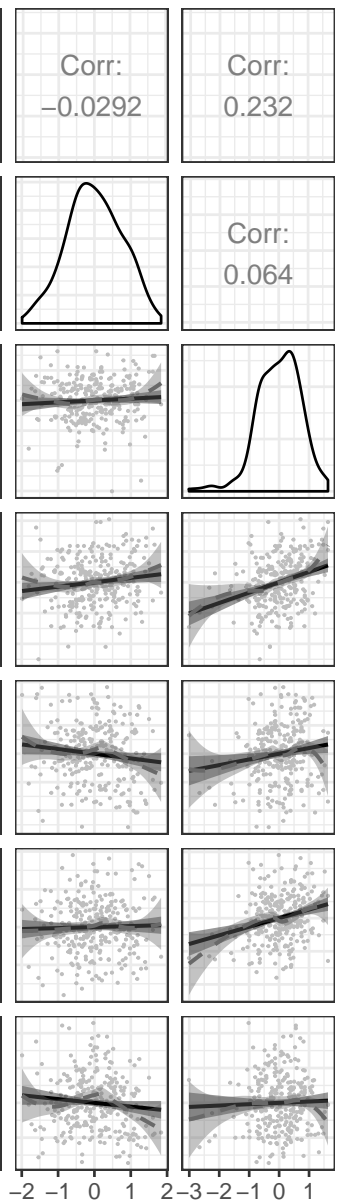
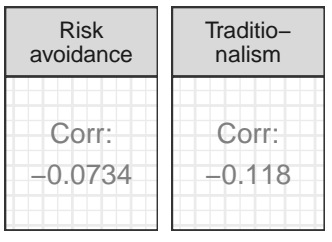

Privacy need

government

Corr:

$-0.139$
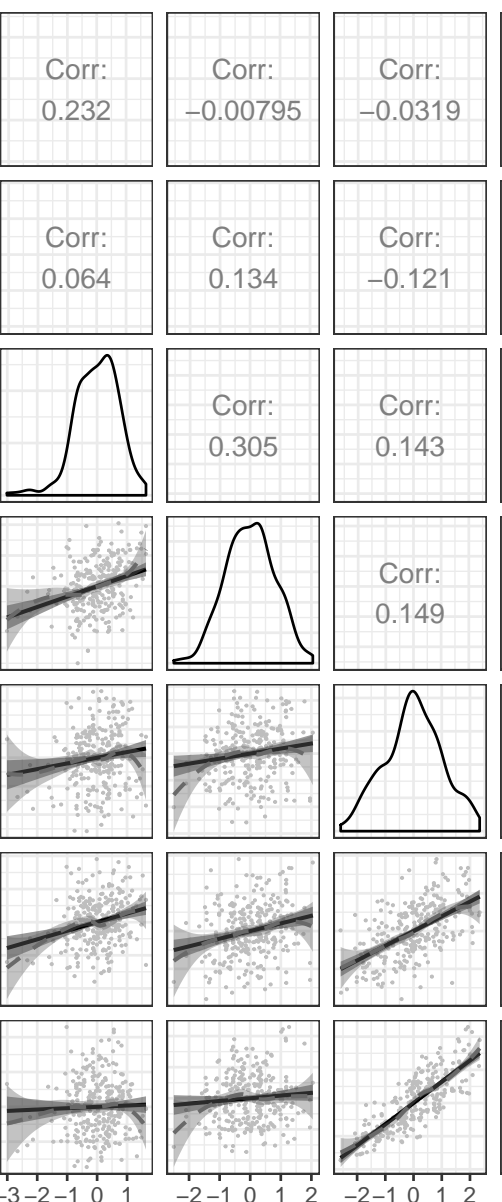
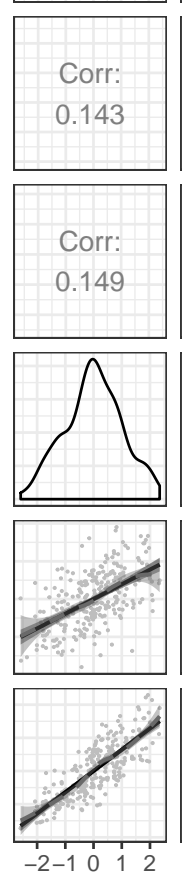
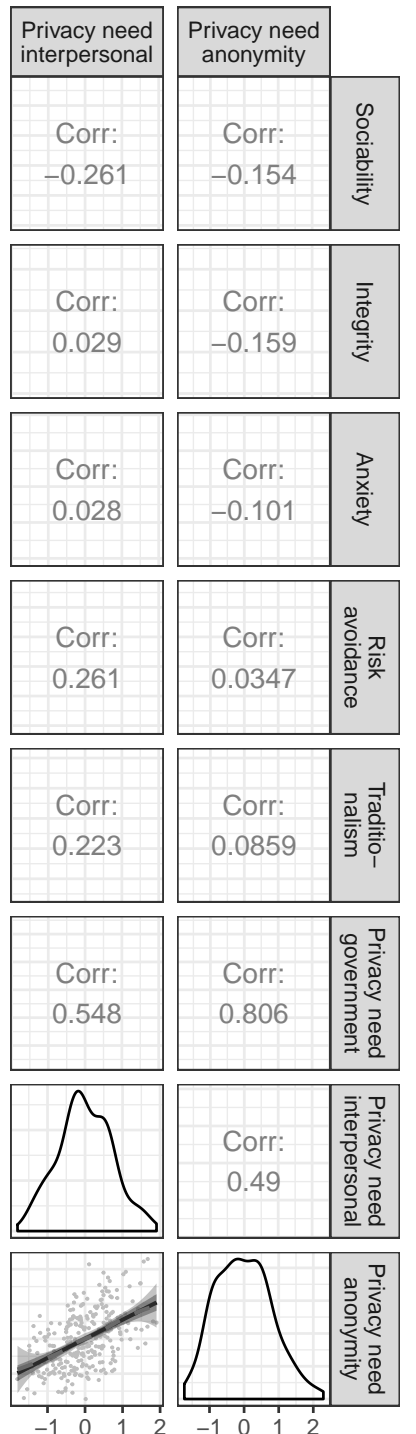

Figure 1. Results of the bivariate relations. Above diagonal: zero-order correlation matrix; diagonal: density plots for each variable; below diagonal: bivariate scatter plots for zeroorder correlations. Solid regression lines represent linear regressions, dashed regression lines represent quadratic regressions. Calculated with the variables' latent factor scores.

The bivariate correlations revealed that respondents who were more sociable than others also needed considerably less privacy from other people. In addition, they also needed slightly less privacy from the government and less anonymity. Also when analyzed 

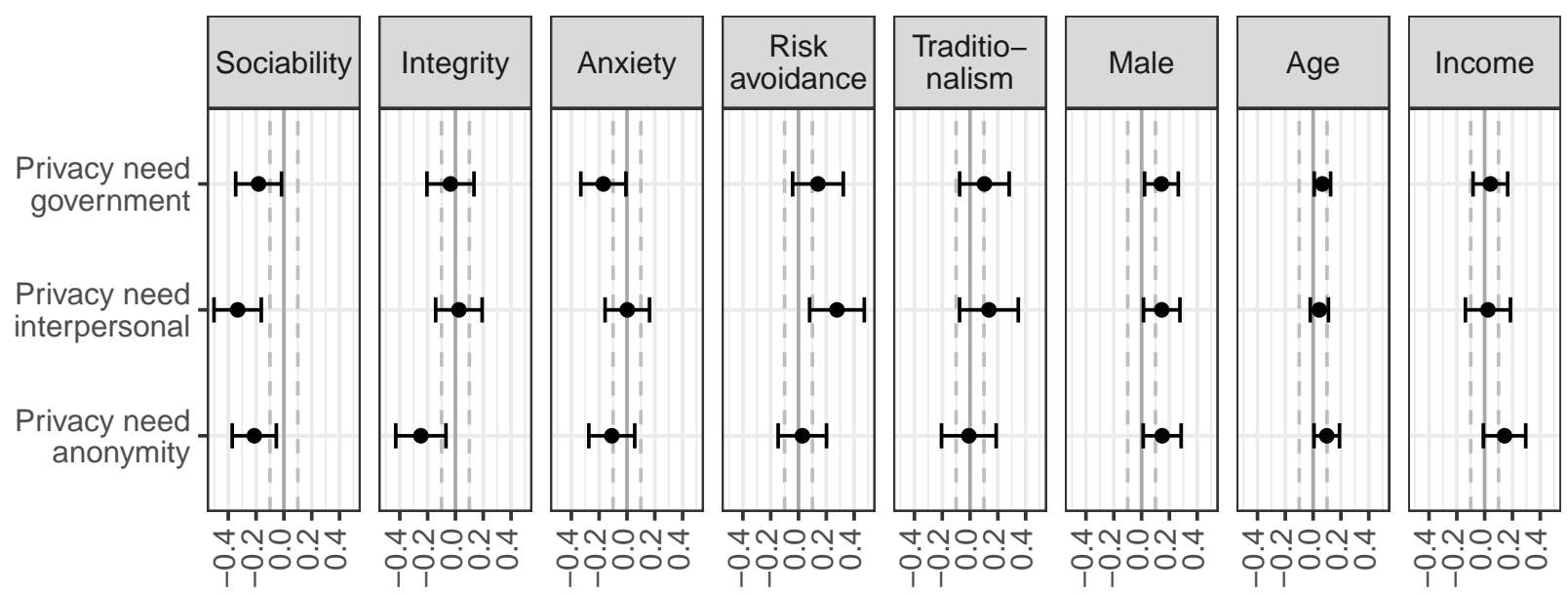

Figure 2. Graphical overview of the results of Structural Equation Model. Shows the $95 \%$ confidence intervals (CIs) of standardized coefficients. (Standard errors were extracted from Lavaan's standardized solution. For the results of a bootstrap approach with 2,000 draws, see OSM.) Dashed lines indicate the SESOI of |.10|.

in the multivariate structural regression model holding all other predictors constant, sociability was related to a reduced need for privacy on all three dimensions.

Next, both the bivariate and the SEM results showed that respondents who reported less integrity compared to others desired considerably more anonymity. No significant relations existed with the need for privacy from the government and the need for anonymity.

For anxiety, both analyses showed that respondents who indicated being more anxious indeed desired less privacy from the government. The effect size was small to moderate. Whereas the bivariate analyses revealed a small significant relation between anxiety and need for anonymity, this relation disappeared when analyzed in the SEM keeping all other predictors constant. No significant relationship with need for privacy from other people emerged.

Both analyses showed that respondents who reported being more risk avoidant than others desired considerably more privacy from other people. The bivariate analyses showed 
a small and positive association between risk avoidance and need for privacy from government surveillance, which ceased to exist in the SEM. No significant relation with need for anonymity was found.

Next, the bivariate analyses showed that respondents who scored higher on traditionalism also reported increased levels of need for privacy from government surveillance and other people. However, both relations disappeared in the SEM. No significant relation with need for anonymity was found.

Finally, regarding socio-demographic variables, the SEM showed that respondents who were older also desired slightly more anonymity. Male respondents desired more privacy from the government and more anonymity. Respondents with a higher income did not exhibit different levels of need for privacy.

Summarizing across the results, the predictors explained $11.84 \%$ of the variance in need for privacy from the government, $29.76 \%$ of the variance in need for privacy from other people, and $17.32 \%$ of the variance in need for anonymity. All predictors were also above our predefined SESOI of $|\beta|=.10$, supporting their theoretical relevance. In addition, note that several nonsignificant effects were actually larger than the predefined SESOI (e.g., the potential effect of risk avoidance on the need for privacy from the government). Hence, it is plausible that a larger study, which yields smaller standard errors, would find additional effects.

As stated above, because all predictors were included on the basis of specific a priori rationales we did not control for alpha error inflation. To better assess whether the results could also be explained by chance alone, let us briefly consider that if the null hypotheses had been true, given the predefined alpha level of $5 \%$ and the 24 statistical tests that were run, one would have expected to find 1.20 statistically significant results by chance alone. In this study we found 11 statistically significant results, which is substantially higher. Similarly, the average $p$-value $p=.28$ was substantially smaller than the average $p$-value of $p=.50$ that one would have expected to find if the null hypotheses had been true. 
Table 3

Results of Structural Equation Model.

\begin{tabular}{|c|c|c|c|c|c|}
\hline Predictor & $\mathrm{b}$ & 11 & $\mathrm{ul}$ & beta & $\mathrm{p}$ \\
\hline \multicolumn{6}{|c|}{ Privacy need government } \\
\hline Sociability & -0.24 & -0.46 & -0.03 & -.18 & .027 \\
\hline Integrity & -0.06 & -0.36 & 0.23 & -.04 & .679 \\
\hline Anxiety & -0.27 & -0.53 & -0.01 & -.17 & .042 \\
\hline Traditionalism & 0.16 & -0.12 & 0.44 & .10 & .255 \\
\hline Risk avoidance & 0.24 & -0.08 & 0.55 & .14 & .142 \\
\hline Male & 0.34 & 0.04 & 0.64 & .14 & .025 \\
\hline Age & 0.03 & $<0.01$ & 0.05 & .07 & .027 \\
\hline Income & 0.04 & -0.09 & 0.18 & .04 & .518 \\
\hline \multicolumn{6}{|c|}{ Privacy need interpersonal } \\
\hline Sociability & -0.38 & -0.57 & -0.19 & -.33 & $<.001$ \\
\hline Integrity & 0.04 & -0.21 & 0.29 & .02 & .770 \\
\hline Anxiety & $<0.01$ & -0.21 & 0.22 & $<.01$ & .977 \\
\hline Traditionalism & 0.18 & -0.11 & 0.47 & .14 & .215 \\
\hline Risk avoidance & 0.40 & 0.13 & 0.67 & .28 & .004 \\
\hline Male & 0.30 & 0.03 & 0.57 & .14 & .031 \\
\hline Age & 0.02 & -0.01 & 0.04 & .04 & .184 \\
\hline Income & 0.02 & -0.13 & 0.17 & .02 & .775 \\
\hline \multicolumn{6}{|c|}{ Privacy need anonymity } \\
\hline Sociability & -0.24 & -0.43 & -0.06 & -.21 & .011 \\
\hline Integrity & -0.37 & -0.63 & -0.10 & -.25 & .008 \\
\hline Anxiety & -0.15 & -0.37 & 0.07 & -.11 & .194 \\
\hline Traditionalism & -0.01 & -0.27 & 0.25 & -.01 & .931 \\
\hline Risk avoidance & 0.04 & -0.21 & 0.29 & .03 & .755 \\
\hline Male & 0.30 & 0.02 & 0.59 & .15 & .037 \\
\hline Age & 0.03 & $<0.01$ & 0.07 & .10 & .036 \\
\hline Income & 0.13 & -0.01 & 0.27 & .14 & .076 \\
\hline
\end{tabular}




\section{Discussion}

This study analyzed the extent to which the need for privacy can be predicted and explained by personality traits. First, the results of the bivariate analyses showed that the need for privacy can be predicted surprisingly well by means of specific personality facets. Second, also when analyzed together in a structural regression model alongside additional socio-demographic variables, most of these personality facets remained significant, supporting the robustness of the relations.

Most prominently, we find that sociability was negatively related to all three dimensions of need for privacy. This does not come to much surprise as privacy captures the withdrawal from others and is hence already from a conceptual point of view closely related to sociability. Nonetheless, it is interesting to see that the relation is indeed thus proximal - especially in light of prior studies not having found a significant relation between extraversion and concern for privacy (Junglas et al., 2008), which are both related variables.

Next, and more controversial, our results also showed that the desire for anonymity is related substantially with measures of integrity. People who self-reported lower integrity desired more anonymity. Respondents who said, for example, that they would feel tempted to take things that do not belong to them were also more likely to avoid situations in which they were identifiable. This finding hence supports the logic behind the "nothing to hide" argument. It also follows Altman's privacy regulation theory (1976), which states that if exposure of information is risky it is likely that people will use more mechanisms to strengthen their social boundaries and increase their desired level of privacy. Importantly, however, integrity was not related to need for privacy from other people or to need for privacy from government surveillance, which hence limits the generalizability of the nothing-to-hide argument. In addition, note that the responses to the items measuring need for anonymity were much lower compared to the other two dimensions of need for privacy (i.e., $M_{\text {anonymity }}=2.92, M_{\text {government }}=4.13, M_{\text {interpersonal }}=4.21$ ). Hence, the 
relationship between integrity and need for privacy might show only in very specific domains that are related to anonymity (for example, avoiding places with video surveillance). In conclusion, the relation between integrity and need for privacy seems to be limited to certain aspects of need for privacy only. It should thus not be overly generalized. Interestingly, the data showed that people who were more anxious were also more willing to accept government surveillance. This might be explained by the fact that governments are explicitly commissioned to help prevent crime or terrorist attacks, which are things that more anxious people are more likely to fear.

People who were more risk averse desired more privacy from other people. Specifically, people the data show that who try to avoid risks are less inclined to share secrets with friends. This finding makes sense and can be aligned with prospect theory (Kahneman \& Tversky, 1979): The risks of a secret being publicly disclosed seem to outweigh the potential benefits of sharing it with confidants among risk-averse people.

Finally, in the SEM no significant relations between need for privacy and traditionality were found. The results imply that independent from being more or less open to change, people desire the same level of privacy. Taken further, this finding might illustrate that people who are more open to change, who are more likely to use novel devices, media, and services, or who might consider themselves as early adopters, do not need less privacy.

At a larger level, the results of this study highlight the importance of making differentiated claims on why people need privacy. Indeed, while the results suggest that some people might need anonymity because they may have something to hide, they also show that putting everyone who exhibits an increased need for privacy under suspicion is wrong. People who are less sociable, more risk averse, and less anxious are also more likely to feel they need more privacy. This implies that various personality-related aspects can predict need for privacy in different ways and for different reasons. 
first to explicitly distinguish different types of privacy needs. Specifically, we found that need for privacy consists of three separate dimensions, with factors measuring need for privacy from the government, need for privacy from other people, and need for anonymity. This factor solution is in line with privacy theory, which subsumes a vertical level (here, privacy from the government) and a horizontal level (here, privacy from other people) (e.g., Schwartz, 1968). The third dimension, need for anonymity, can be argued to exist on the diagonal, as one can be anonymous both from the government and from other people. The results of our study demonstrate the importance of differentiating these types of need for privacy, as relationships were not consistent across the various privacy needs and the five personality traits.

\section{Limitations and Future Research}

One can of course raise the question of whether it is possible or even socially desirable to measure a person's integrity. On the one hand, integrity implies absolute criteria in relation to social norms: Stealing is bad and forbidden, whereas helping is good and encouraged. On the other hand, integrity implies relative criteria: Whereas some cultures disapprove of lying whatever the context, others consider lying okay in some situations (for example, "white lies" in order to avoid hurting someone's feelings) (Altman, 1977). Thus, ranking behaviors, opinions, and character traits with regard to integrity presents a moral dilemma. As a result, throughout our study we have employed a conservative approach to understand and measure integrity as an explicit transgression of social norms that is strong and that most societies would arguably agree upon (for example, most societies would consider law-breaking as a sign of low integrity). Nonetheless, we recommend that future research should further elaborate on the general understanding of integrity as a theoretical concept. To date, there is not one overarching concept of integrity that incorporates all of the different aspects of this variable, and yet it would be valuable to examine how other aspects of integrity (e.g., authenticity, 
trustworthiness, or consistency) relate to need for privacy.

Worth nothing, integrity tests using self-reports have been shown to work surprisingly well, given that they can predict unwanted professional workplace behavior successfully (e.g., theft, drug and alcohol problems, or absenteeism) (Ones, Viswesvaran, \& Schmidt, 1993). In a meta-analysis with 665 correlation coefficients, self-reported integrity tests were associated with counterproductive behaviors with an average correlation coefficient of $r=$ .47 (Ones et al., 1993). Nonetheless, future research would benefit from including behavioral manifestations of integrity, such as concrete cheating behaviors.

Another limitation of the study is that despite using mostly well-established scales, confirmatory factor analyses revealed that most measures did not show satisfactory factorial validity, which resembles the current finding and criticism by Hussey and Hughes (2018). In order to achieve sufficient factorial validity we therefore needed to adapt the scales (for example by deleting items). However, this introduces problems of overfitting, which in turn potentially impedes the generalizability of the results (see, e.g., Yarkoni \& Westfall, 2017). By using bifactor models we explicitly tried to retain the largest possible number of items, which should render our results more robust. In addition, also when looking at the items individually (see the OSM) one can see that the results are not overly dependent on inclusion of specific items.

Another methodological improvement for future studies concerns the sample, which was comparatively small, leading to a reduced power to detect also small effects. Nonetheless, we are still confident that the results should be reliable because we deliberately selected specific predictors that should exhibit at least small to moderate relations with need for privacy (i.e., $r=.20$ ). For small to moderate relations, the current study still had a convincing power of $92 \%$. Granted, when aiming to find also small effects (i.e., $r=.10$ ) with a probability of $95 \%$, future studies would need to collect data from 1293 respondents (Cohen, 1992). Finally, generalizability of our sample is limited, as it was predominantly young, female, highly educated, and collected only in one country. 
Finally, future research might consider analyzing predictors of privacy needs that are even more specified. For example, it is possible that people who hold dissenting political beliefs could also perceive a higher need for privacy from the government. In this study we focused mostly on escapist motives for why people desire privacy (e.g., sociability, risk aversion). Interestingly, Leary, Herbst, and McCrary (2003) showed that when predicting engagement in solitary activities, it is less preferable to measure how strongly people want to escape society (avoidance oriented), but rather how much they seek solitude (approach oriented). Hence, future studies might want to include predictors such as need for contemplation or trust (see, e.g., Metzger, 2004; Wheeless \& Grotz, 1977). Finally, it would be interesting to focus on need for privacy within different minority groups. For example, it seems plausible that people with a LGBT background might report needing more privacy from government (because it is potentially repressive or unfriendly toward LGBTs) or other people (especially weaker ties in their social networks who might not approve of their sexual identity).

\section{Conclusion}

Privacy is getting more impinged on these days with digital surveillance and intense social pressure toward self-disclosure via social networks, which have both negative and positive aspects at the same time. In this context it is understandable why people might vary in their felt need for privacy. Our study sheds light on what drives that variation, finding that sociability, and to a lesser extent, anxiety, risk aversion, and integrity all contribute to individuals' need for privacy, albeit in different ways. 


\section{References}

Altman, I. (1975). The environment and social behavior. Monterey, CA: Brooks Cole.

Altman, I. (1976). Privacy: A conceptual analysis. Environment and Behavior, 8(1), 7-29. doi:10.1177/001391657600800102

Altman, I. (1977). Privacy regulation: Culturally universal or culturally specific? Journal of Social Issues, 33(3), 66-84. doi:10.1111/j.1540-4560.1977.tb01883.x

Aust, F., \& Barth, M. (2018). papaja: Create APA manuscripts with R Markdown. Retrieved from https://github.com/crsh/papaja

Bansal, G., Zahedi, F. M., \& Gefen, D. (2010). The impact of personal dispositions on information sensitivity, privacy concern and trust in disclosing health information online. Decision Support Systems, 49(2), 138-150. doi:10.1016/j.dss.2010.01.010

Bol, N., Dienlin, T., Kruikemeier, S., Sax, M., Boerman, S. C., Strycharz, J., .. Vreese, C. H. de. (2018). Understanding the effects of personalization as a privacy calculus: Analyzing self-disclosure across health, news, and commerce contexts. Journal of Computer-Mediated Communication, 23(6), 370-388. doi:10.1093/jcmc/zmy020

boyd, danah m. (2008). Taken out of context. American teen sociality in networked publics: Doctoral dissertation. Berkeley, CA: University of California.

Burgoon, J. K. (1982). Privacy and communication. Annals of the International Communication Association, 1, 206-249.

Buss, A. H. (2001). Psychological dimensions of the self. Thousand Oaks; Calif: Sage Publications.

Champely, S. (2018). Pwr: Basic functions for power analysis. Retrieved from https://CRAN.R-project.org/package $=$ pwr

Cohen, J. (1992). A power primer. Psychological Bulletin, 112(1), 155-159. doi:10.1037/0033-2909.112.1.155

Connelly, S., Lilienfeld, S. O., \& Schmeelk, K. M. (2006). Integrity tests and morality: Associations with ego development, moral reasoning, and psychopathic personality. 
International Journal of Selection and Assessment, 14(1), 82-86.

doi:10.1111/j.1468-2389.2006.00335.x

Corcoran, K. J., \& Rotter, J. B. (1987). Morality-conscience guilt scale as a predictor of ethical behavior in a cheating situation among college females. The Journal of General Psychology, 114(2), 117-123. doi:10.1080/00221309.1987.9711061

Costa, P. T., \& McCrae, R. R. (1992). Normal personality assessment in clinical practice: The NEO Personality Inventory. Psychological Assessment, 4(1), 5-13. doi:10.1037/1040-3590.4.1.5

Costello, A. B., \& Osborne, J. W. (2005). Best practices in exploratory factor analysis: Four recommendations for getting the most from your analysis. Practical Assessment, Research \& Evaluation, 7(10). Retrieved from http://pareonline.net/pdf/v10n7.pdf

Covey, M. K., Saladin, S., \& Killen, P. J. (1989). Self-monitoring, surveillance, and incentive effects on cheating. The Journal of Social Psychology, 129(5), 673-679. doi:10.1080/00224545.1989.9713784

Dienlin, T. (2014). The privacy process model. In S. Garnett, S. Halft, M. Herz, \& J. M. Mönig (Eds.), Medien und Privatheit (pp. 105-122). Passau, Germany: Karl Stutz.

Dienlin, T., \& Metzger, M. J. (2016). An extended privacy calculus model for SNSs-Analyzing self-disclosure and self-withdrawal in a representative U.S. sample. Journal of Computer-Mediated Communication, 21(5), 368-383. doi:10.1111/jcc4.12163

Donnellan, M. B., \& Lucas, R. E. (2008). Age differences in the Big Five across the life span: Evidence from two national samples. Psychology and Aging, 23(3), 558-566. doi:10.1037/a0012897

Fife, E., \& Orjuel, J. (2012). The privacy calculus: Mobile apps and user perceptions of privacy and security. International Journal of Engineering Business Management, 4, 1-10. doi:10.5772/51645 
Granovetter, M. S. (1973). The strength of weak ties. American Journal of Sociology, $78(6), 1360-1380$.

Greenwald, G. (2013). NSA collecting phone records of millions of Verizon customers daily. The Guardian. Retrieved from www.theguardian.com

Hussey, I., \& Hughes, S. (2018). Hidden invalidity among fifteen commonly used measures in social and personality psychology. doi:10.31234/osf.io/7rbfp

John, O. P., \& Srivastava, S. (1999). The big five trait taxonomy: History, measurement, and theoretical perspectives. In L. A. Pervin \& O. P. John (Eds.), Handbook of personality (pp. 102-138). New York, NY: Guilford Press.

Johnson, B. (2010). Privacy no longer a social norm, says Facebook founder. The Guardian. Retrieved from www.theguardian.com

Jorgensen, D., T., Pornprasertmanit, S., Schoemann, M., A., .. Y. (2018). sem Tools: Useful tools for structural equation modeling. Retrieved from https://CRAN.R-project.org/package=semTools

Junglas, I. A., Johnson, N. A., \& Spitzmüller, C. (2008). Personality traits and concern for privacy: an empirical study in the context of location-based services. European Journal of Information Systems, 17(4), 387-402. doi:10.1057/ejis.2008.29

Kahneman, D., \& Tversky, A. (1979). Prospect theory: An analysis of decision under risk. Econometrica, 47(2), 263. doi:10.2307/1914185

Kline, R. B. (2016). Principles and practice of structural equation modeling (4th ed.). New York, NY: The Guilford Press.

Korkmaz, S., Goksuluk, D., \& Zararsiz, G. (2014). MVN: An r package for assessing multivariate normality. The $R$ Journal, 6(2), 151-162. Retrieved from https://journal.r-project.org/archive/2014-2/korkmaz-goksuluk-zararsiz.pdf

Lakens, D., Scheel, A. M., \& Isager, P. M. (2018). Equivalence testing for psychological research: A tutorial. Advances in Methods and Practices in Psychological Science, 1(2), 259-269. doi:10.1177/2515245918770963 
Leary, M. R., Herbst, K. C., \& McCrary, F. (2003). Finding pleasure in solitary activities: Desire for aloneness or disinterest in social contact? Personality and Individual Differences, 35(1), 59-68. doi:10.1016/S0191-8869(02)00141-1

Masur, P. K. (2018). Situational privacy and self-disclosure: Communication processes in online environments. Cham, Switzerland: Springer.

Meijer, R. R., Niessen, A. S. M., \& Tendeiro, J. N. (2016). A practical guide to check the consistency of item response patterns in clinical research through person-fit statistics: Examples and a computer Program. Assessment, 23(1), 52-62. doi:10.1177/1073191115577800

Metzger, M. J. (2004). Privacy, trust, and disclosure: Exploring barriers to electronic commerce. Journal of Computer-Mediated Communication, 9(4). doi:10.1111/j.1083-6101.2004.tb00292.x

Morton, A. (2013). Measuring inherent privacy concern and desire for privacy - A pilot survey study of an instrument to measure dispositional privacy concern. In International Conference on Social Computing (SocialCom) (pp. 468-477). doi:10.1109/SocialCom.2013.73

Nissenbaum, H. (2010). Privacy in context: Technology, policy, and the integrity of social life. Stanford, CA: Stanford University Press.

Omarzu, J. (2000). A disclosure decision model: Determining how and when individuals will self-disclose. Personality and Social Psychology Review, 4(2), 174-185. doi:10.1207/S15327957PSPR0402_5

Ones, D. S., Viswesvaran, C., \& Schmidt, F. L. (1993). Comprehensive meta-analysis of integrity test validities: Findings and implications for personnel selection and theories of job performance. Journal of Applied Psychology, 78(4), 679-703. doi:10.1037/0021-9010.78.4.679

Park, Y. J. (2015). Do men and women differ in privacy? Gendered privacy and (in)equality in the Internet. Computers in Human Behavior, 50, 252-258. 
doi:10.1016/j.chb.2015.04.011

Paunonen, S. V. (2002). Design and construction of the Supernumerary Personality Inventory. London, Canada: University of Western Ontario.

Paunonen, S. V., \& Ashton, M. C. (2001). Big Five factors and facets and the prediction of behavior. Journal of Personality and Social Psychology, 81(3), 524-539. doi:10.1037/0022-3514.81.3.524

Pedersen, D. M. (1979). Dimensions of privacy. Perceptual and Motor Skills, 48(3), 1291-1297. doi:10.2466/pms.1979.48.3c.1291

Pedersen, D. M. (1982). Personality correlates of privacy. The Journal of Psychology, 112(1), 11-14. doi:10.1080/00223980.1982.9923528

Petronio, S. (2010). Communication privacy management theory: What do we know about family privacy regulation? Journal of Family Theory $\&$ Review, 2(3), 175-196. doi:10.1111/j.1756-2589.2010.00052.x

Pew Research Center. (2015). Beyond distrust: How Americans view their government. Retrieved from http://www.people-press.org/2015/11/23/ beyond-distrust-how-americans-view-their-government/

Pew Research Center. (2017). Public trust in government: 1958-2017. Retrieved from http://www.people-press.org/2017/12/14/public-trust-in-government-1958-2017/

R Core Team. (2018). R: A language and environment for statistical computing. Vienna, Austria: R Foundation for Statistical Computing. Retrieved from https://www.R-project.org/

Revelle, W. (2018). Psych: Procedures for psychological, psychometric, and personality research. Evanston, Illinois: Northwestern University. Retrieved from https://CRAN.R-project.org/package=psych

Rosseel, Y. (2012). lavaan: An R package for structural equation modeling. Journal of Statistical Software, 48(2), 1-36. Retrieved from http://www.jstatsoft.org/v48/i02/

Schloerke, B., Crowley, J., Cook, D., Briatte, F., Marbach, M., Thoen, E., ... Larmarange, 
J. (2018). GGally: Extension to 'ggplot2'. Retrieved from https://CRAN.R-project.org/package=GGally

Schwartz, B. (1968). The social psychology of privacy. American Journal of Sociology, $73(6), 741-752$.

Sevignani, S. (2016). Privacy and capitalism in the age of social media. New York; London: Routledge Taylor \& Francis Group.

Sheldon, K. M. (2004). Integrity [authenticity, honesty]. In C. Peterson \& Seligman, M. E. P. (Eds.), Character strengths and virtues: A handbook and classification (pp. 249-271). Oxford, UK: Oxford University Press.

Solove, D. J. (2007). 'I've got nothing to hide' and other misunderstandings of privacy. San Diego Law Review, 44, 745-772.

Stone, D. L. (1986). Relationship between introversion/extraversion, values regarding control over information, and perceptions of invasion of privacy. Perceptual and Motor Skills, 62(2), 371-376. doi:10.2466/pms.1986.62.2.371

Tendeiro, J. N., Meijer, R. R., \& Niessen, A. S. M. (2016). PerFit: An R package for person-fit analysis in IRT. Journal of Statistical Software, 74(5), 1-27. doi:10.18637/jss.v074.i05

Tifferet, S. (2019). Gender differences in privacy tendencies on social network sites: A meta-analysis. Computers in Human Behavior, 93, 1-12. doi:10.1016/j.chb.2018.11.046

Trepte, S., Dienlin, T., \& Reinecke, L. (2013). Privacy, self-disclosure, social support, and social network site use. Research report of a three-year panel study. Retrieved from http://opus.uni-hohenheim.de/volltexte/2013/889/

Trepte, S., \& Masur, P. K. (2017). Need for privacy. In V. Zeigler-Hill \& T. K. Shackelford (Eds.), Encyclopedia of Personality and Individual Differences (Vol. 94, pp. 1-4). Cham: Springer International Publishing. doi:10.1007/978-3-319-28099-8_540-1 van Buuren, S., \& Groothuis-Oudshoorn, K. (2011). mice: Multivariate imputation by 
chained equations in r. Journal of Statistical Software, 45(3), 1-67. Retrieved from https://www.jstatsoft.org/v45/i03/

Weinberger, M., Zhitomirsky-Geffet, M., \& Bouhnik, D. (2017). Sex differences in attitudes towards online privacy and anonymity among Israeli students with different technical backgrounds, 22(4). Retrieved from http://InformationR.net/ir/22-4/paper777.html

Westin, A. F. (1967). Privacy and freedom. New York, NY: Atheneum.

Wheeless, L. R., \& Grotz, J. (1977). The measurement of trust and its relationship to self-disclosure. Human Communication Research, 3(3), 250-257. doi:10.1111/j.1468-2958.1977.tb00523.x

Wickham, H. (2016). Ggplot2: Elegant graphics for data analysis. Springer-Verlag New York. Retrieved from http://ggplot2.org

Wickham, H. (2017). Tidyverse: Easily install and load the 'tidyverse'. Retrieved from https://CRAN.R-project.org/package=tidyverse

Xie, Y. (2015). Dynamic documents with $R$ and knitr (2nd ed.). Boca Raton, Florida: Chapman; Hall/CRC. Retrieved from https://yihui.name/knitr/

Yarkoni, T., \& Westfall, J. (2017). Choosing prediction over explanation in psychology: Lessons from machine learning. Perspectives on psychological science : a journal of the Association for Psychological Science, 12(6), 1100-1122. doi:10.1177/1745691617693393 
- Contributed to conception and design: TD, MM

- Contributed to acquisition of data: MM, TD

- Contributed to analysis and interpretation of data: TD, MM

- Drafted and/or revised the article: TD, MM

- Approved the submitted version for publication: TD, MM

\section{Funding Information}

During the conception and data collection of the study, TD was funded by The German Academic Scholarship Foundation (German: Studienstiftung des deutschen Volkes), which financially supported TD's research stay at UCSB. During the writing of the article, TD was funded by the Volkswagen Foundation (German: Volkswagenstiftung), grant "Transformations of Privacy".

MM is funded by a regular and tenured full professorship at UCSB.

\section{Competing Interests}

We declare no competing interests.

\section{Data Accessibility Statement}

All the stimuli, presentation materials, participant data, analysis scripts, and a reproducible version of the manuscript can be found in the Online Supplementary Material on the open science framework (https://osf.io/7ncpk/). In addition, we invite everyone to suggest changes and improvements to the manuscript via our github (https://github.com/tdienlin/need_for_privacy/). 